\title{
AN EXACT RANK TEST FOR SCALE UNDER NORMALITY USING HELMERT'S TRANSFORMATION
}

\author{
Willem ALBERS* \\ Twente University of Technology, Enschede, The Netherlands
}

Received 6 November 1983; revised manuscript received 13 February 1985

Recommended by M.L. Puri

\begin{abstract}
In the problem of testing equality of scale of two distributions a rank test should be preferred over the $F$-test if it is not sure that the distributions involved are normal. However, if in addition the distributions may also differ in location, it becomes necessary to first adjust the observations, and the rank test will then at best be asymptotically distribution-free, even if normality holds after all. In this paper it is demonstrated how using Helmert's transformation for the adjustment of the observations leads to a rank test which is exact under normality and asymptotically distribution-free otherwise.
\end{abstract}

AMS Subject Classification: 62G10, 62G20.

Key words: Two-sample scale problem; Rank test; Helmert's transformation; Nuisance parameters.

\section{Introduction}

Let $X_{1}, \ldots, X_{m}$ and $X_{m+1}, \ldots, X_{n}$ be two samples from continuous distribution functions (df's) $F((x-\mu) / \sigma)$ and $F((x-v) / \tau)$, respectively, where $\mu, \nu, \sigma$ and $\tau$ are unknown parameters. Suppose we are interested in testing the hypothesis $\mathrm{H}_{0}$ that the scale parameters $\sigma$ and $\tau$ are equal. First assume that $\mu=\nu$. If moreover we can assume that $F=\Phi$, where $\Phi$ stands for the standard normal df, then the classical $F$-test can be applied. However, it is well known that if there is any doubt about this normality assumption, the $F$-test, which is not even asymptotically distributionfree, should not be used (see e.g. Lehmann (1975), p. 102). In that case a rank test, which is distribution-free for $\mathrm{H}_{0}$, should be preferred. Its statistic can be written as

$$
W_{N}^{*}=\sum_{j=m+1}^{N} a_{N}\left(R_{j}\right)
$$

* Presently at University of Limburg, Maastricht, The Netherlands. 
in which $R_{1}, \ldots, R_{N}$ are the ranks of $\left(X_{1}, \ldots, X_{N}\right)$ and the $a_{N}(i)$ are either the exact or the approximate scores generated from a function $J$ on $(0,1)$, that is

$$
a_{N}(i)=E J\left(U_{i: N}\right) \quad \text { or } \quad a_{N}(i)=J\left(\frac{i}{N+1}\right),
$$

where $U_{1: N}<\cdots<U_{N: N}$ are order statistics of a sample of size $N$ from the uniform distribution on $(0,1)$. For given $F$, the best choice of $J$ is (cf. Hájek and Šidák (1967), p. 69)

$$
J_{F}(t)=-1-F^{-1}(t) f^{\prime}\left(F^{-1}(t)\right) / f\left(F^{-1}(t)\right),
$$

where $f^{\prime}$ is the derivative of the density $f$ of $F$ and $F^{-1}$ is the inverse of $F$. For $F=\Phi$ we can use $J_{F}=\left(\Phi^{-1}\right)^{2}$, which leads through (1.2) to the Capon and Klotz tests, respectively. These tests have asymptotic relative efficiency (ARE) 1 with respect to the $F$-test under normality.

Next we drop the assumption that $\mu=\nu$. For the classical case it is well known how to overcome this frequently occurring complication: replace $\mu$ and $\nu$ by the corresponding sample means and apply the $F$-test with $(m-1, N-m-1)$ rather than with $(m, N-m)$ degrees of freedom. For the rank test case, the situation is somewhat less simple. Obviously, here the natural thing to do also is to replace the original random variables (rv's) $X_{j}$ by adjusted rv's

$$
\tilde{X}_{j}= \begin{cases}X_{j}-\tilde{\mu}, & j=1, \ldots, m, \\ X_{j}-\tilde{v}, & j=m+1, \ldots, N,\end{cases}
$$

where $\tilde{\mu}$ and $\tilde{v}$ are appropriate estimators of the nuisance parameters $\mu$ and $\nu$, respectively (see e.g. Lehmann (1975), p. 104). Appropriate here typically means that $m^{1 / 2}(\tilde{\mu}-\mu)$ and $(N-m)^{1 / 2}(\tilde{v}-v)$ are bounded in probability.

However, this operation clearly destroys the distribution-freeness of our rank test. In fact, we cannot even take for granted that the test will be asymptotically distribution-free. Several authors, among others Sukhatme (1958), Raghavachari (1965) and Hájek (1970), have investigated under what conditions at least this last property is preserved. It turns out that it typically suffices to require symmetry of $f$ around 0 and of $J$ around $\frac{1}{2}$. (Note that if $J$ is chosen according to (1.3), the first condition implies the second.) Then the test based on the $\tilde{X}_{j}$ can be shown to be asymptotically equivalent to the (ideal but not available) test based on the $X_{j}-\mu$, $j \leq m$ and the $X_{j}-v, j>m$, which clearly implies that the first test is asymptotically distribution-free. Hence in particular the Capon and Klotz tests based on adjusted rv's still have ARE 1 with respect to the $F$-test under normality and moreover are asymptotically distribution-free under symmetric $f$.

Now we reconsider the situation described above from the following point of view. If we want to persuade someone to use a nonparametric rather than a classical procedure, it is important to impress him by pointing out by the advantage of the nonparametric over the classical procedure if the ideal model is not true. But it is probably even more vital to make clear to him how little he stands to lose if he uses 
the nonparametric procedure and the ideal model happened to be true after all. If we consider the above in this light, then we must conclude that in going from the case of known $\mu$ and $v$ to the case where $\mu$ and $v$ are nuisance parameters, the nonparametric test loses quite a bit of its attractiveness since in the latter situation we no longer have an exact rank test, even if the model is true.

The aim of the present paper is to show how this additional drawback can be removed under the ideal model. The idea is quite simple: the classical approach rests on applying orthonormal transformations to both $\left(X_{1}, \ldots, X_{m}\right)$ and $\left(X_{m+1}, \ldots, X_{N}\right)$, thus producing two new sets of rv's, say $\left(\hat{X}_{1}, \ldots, \hat{X}_{m}\right)$ and $\left(\hat{X}_{m+1}, \ldots, \hat{X}_{N}\right)$. The only requirement involved is that in each of these new sets one of the rv's is proportional to the corresponding sample mean. Choose e.g. $\hat{X}_{1}=m^{-1 / 2} \sum_{i=1}^{m} X_{i}$ and $\hat{X}_{N}=$ $(N-m)^{-1 / 2} \sum_{i=m+1}^{N} X_{i}$. In the classical case it is not necessary to worry about an actual choice for the remaining $\hat{X}_{j}$. It suffices to note that these rv's have to be independent and normal again, with variance $\sigma^{2}$ for $j=2, \ldots, m$ and variance $\tau^{2}$ for $j=m+1, \ldots, N-1$, but with mean 0 (rather than $\mu$ or $v$ ) for all $j$ involved.

This last observation reveals how we can obtain an exact rank test if the normal model is correct: in contradistinction to the classical case, we now do make an explicit choice for $\left(\hat{X}_{2}, \ldots, \hat{X}_{m}\right)$ and $\left(\hat{X}_{m+1}, \ldots, \hat{X}_{N-1}\right)$ and subsequently we base our rank test from (1.1) on these new rv's. Actually such a choice is readily available. It is produced by the so-called Helmert transformation (see Kendall and Stuart (1969), p. 250). In the present situation we can perform this transformation for example as follows:

$$
\begin{aligned}
& \hat{X}_{j}=\varrho_{j}\left(X_{j}-\bar{X}_{j}\right), \\
& \varrho_{j}^{2}=\frac{j-1}{j}, \quad \bar{X}_{j}=(j-1)^{-1} \sum_{i=1}^{j-1} X_{i}, \quad j=2, \ldots, m, \\
& \varrho_{j}^{2}=\frac{N-j}{N-j+1}, \quad \bar{X}_{j}=(N-j)^{-1} \sum_{i=j+1}^{N} X_{i}, \quad j=m+1, \ldots, N-1 .
\end{aligned}
$$

One easily verifies that these $\hat{X}_{j}$ indeed have mean 0 , variance either $\sigma^{2}$ or $\tau^{2}$, and moreover are uncorrelated, which under normality means that these rv's are independent.

Hence by using the adjusted rv's $\hat{X}_{j}$ rather than the $\tilde{X}_{j}$ from (1.4), we have indeed removed the additional disadvantage under the ideal model and it may look as if we have already achieved our goal. Unfortunately, that is not true. It remains to investigate where using the $\hat{X}_{j}$ leads to if the normal model does not hold. More precisely, in the above we noted that under appropriate symmetry conditions the use of the $\bar{X}_{j}$ will produce a test which is asymptotically equivalent to the ideal test based on the $X_{j}-\mu$ or $\nu$. Hence we must demonstrate that the $\hat{X}_{j}$ have a similar property. If this would not be the case, it would mean a disadvantage which clearly outweighs the advantage observed earlier, and it would not make sense to consider using the $\hat{X}_{j}$. 
In the next sections we shall obtain conditions under which the $\hat{X}_{j}$ indeed have the desired property. At this point we merely note that, roughly speaking, the $\hat{X}_{j}$ are more complicated to deal with than the $\tilde{X}_{j}$ in two respects. In the first place, in the $\tilde{X}_{j}$ we do not use the same estimators for $\mu$ and $v$ all the time, but we let these estimators depend on $j$. Moreover, for the $\tilde{X}_{j}$ we typically have that $\tilde{X}_{j}-X_{j}=$ $\mathrm{O}_{P}\left(N^{-1 / 2}\right)$, whereas in case of the $\hat{X}_{j}$ we are faced with rather unpleasant boundary effects as typically $\hat{X}_{j}-X_{j}=\mathrm{O}_{P}\left(j^{-1 / 2}+(N-j)^{-1 / 2}\right)$.

\section{Asymptotic equivalence of the tests involved}

As was observed in the introduction, our task now is to investigate under what circumstances the test based on the $\hat{X}_{j}$ from (1.5) is asymptotically equivalent to the test based on the $X_{j}-\mu, j \leq m$, and the $X_{j}-v, j>m$. Since this will turn out to be a rather technical matter, we shall, besides presenting the main result, restrict ourselves in this section to sketching the approach used and the conditions involved. The details will be collected in the appendix.

To begin with we note that, as both tests are translation invariant, we may without loss of generality assume in the sequel that $\mu=\nu=0$. By a similar argument we may also suppose that $\sigma=1$. Moreover, it will turn out to be convenient to use a slightly more general statistic than $W_{N}^{*}$ from (1.1). To this end we introduce regression constants $c_{j}$ satisfying

$$
\sum_{j=1}^{N} c_{j}=0, \quad \sum_{j=1}^{N} c_{j}^{2}=1, \quad \max _{1 \leq j \leq N}\left|c_{j}\right|=\mathrm{O}\left(N^{-1 / 2}\right) .
$$

Then we consider the rank statistic $W_{N}$ and the standardized ranks $\xi_{j}$ given by

$$
W_{N}=\sum_{j=1}^{N} c_{j} J\left(\xi_{j}\right), \quad \xi_{j}=(N+1)^{-1} \sum_{k=1}^{N} u\left(X_{j}-X_{k}\right),
$$

where $u(x)=1$ for $x \geq 0$ and $u(x)=0$ otherwise. Typically, we will have in mind the choice $c_{j}=-(N-m)^{1 / 2} m^{-1 / 2} N^{-1 / 2}, j \leq m, c_{j}=m^{1 / 2}(N-m)^{-1 / 2} N^{1 / 2}, j>m$, which is appropriate for the two-sample case we are interested in, but we shall not use this explicitly. Merely note that for this choice the third condition in (2.1) is fulfilled if $m / N$ is bounded away from 0 and 1 . As concerns (2.2), note that we take the approximate scores case as our starting point. Later on we shall show that the results obtained also extend to the case of exact scores.

In analogy to (2.2), the statistic $\hat{W}_{N}$ of the test based on the $\hat{X}_{j}$ from (1.5) is now obtained through standardized ranks $\hat{\xi}_{j}$ as follows

$$
\hat{W}_{N}=\sum_{j=2}^{N-1} c_{j} J\left(\hat{\xi}_{j}\right), \quad \hat{\xi}_{j}=(N+1)^{-1} \sum_{k=2}^{N-1} u\left(\hat{X}_{j}-\hat{X}_{k}\right) .
$$

In the next lemma a first set of conditions are given under which the tests based on $\hat{W}_{N}$ from (2.3) and on $W_{N}$ from (2.2) are asymptotically equivalent for testing $\mathrm{H}_{0}$ against contiguous scale alternatives. 
Lemma 2.1. Let $X_{1}, \ldots, X_{m}$ and $X_{m+1}, \ldots, X_{N}$ be independent samples from the $d f s$ $F(x)$ and $F(x /(1+\theta))$, respectively, where $0 \leq \theta=\mathrm{O}\left(N^{-1 / 2}\right)$ and $m / N$ is bounded away from 0 and 1 . Suppose that the $c_{j}$ satisfy (2.1) and that $J$ from (2.2) and $J_{F}$ from (1.3) are square-integrable. Then

$$
E_{0}\left|\hat{W}_{N}-W_{N}\right|=o(1)
$$

implies that

$$
\sup _{x}\left|P_{\theta}\left(\hat{W}_{N} \leq x\right)-P_{\theta}\left(W_{n} \leq x\right)\right|=\mathrm{o}(1),
$$

where $E_{\theta}$ and $P_{\theta}$ stand for expectation and probability under $\theta$.

Proof. Markov's inequality and (2.4) imply that $\hat{W}_{N}-W_{N} \rightarrow 0$ in $P_{0}$-probability. As $J_{F}$ is square-integrable, $\theta=\mathrm{O}\left(N^{-1 / 2}\right)$, and $m / N$ is bounded away from 0 and 1 , we have contiguity and therefore $\hat{W}_{N}-W_{N} \rightarrow 0$ also holds in $P_{\theta}$-probability for $\theta>0$. From this result (2.5) follows by Slutsky's theorem (see e.g. Cramér (1946), p. 254).

Note that the assumptions above on $\theta, m / N, J, J_{F}$ and the $c_{j}$ are standard conditions which are imposed when the test based on $W_{N}$ is used for testing $\mathrm{H}_{0}$ against contiguous alternatives. Hence the only additional condition involved is (2.4) and it remains to verify when this will hold. In doing so, we will be working under $\mathrm{H}_{0}$ all the time and therefore we shall in the sequel simply write $E$ and $P$ instead of $E_{0}$ and $P_{0}$.

In the present situation it seems indicated to attack $\hat{W}_{N}-W_{N}$ by applying an expansion of the type introduced by Hájek (1968) for the case of $W_{N}-V_{N}$, where

$$
V_{N}=\sum_{j=1}^{N} c_{j} J\left(\Gamma_{j}\right), \quad \Gamma_{j}=F\left(X_{j}\right) .
$$

Since $E\left(W_{N}-V_{N}\right)^{2}=o(1)$ under the conditions of Lemma 2.1 (cf. Hájek and Šidák (1967), p. 161), we can also consider the expansion for $\hat{W}_{N}-V_{N}$, rather than the one for $\hat{W}_{N}-W_{N}$. It will basically look like $\hat{W}_{N}-V_{N}=\bar{Q}_{N}+\frac{1}{2} \bar{R}_{N}$, in which

$$
\begin{aligned}
& \bar{Q}_{N}=\sum_{j=2}^{N-1} c_{j} J^{\prime}\left(\Gamma_{j}\right)\left(\hat{\xi}_{j}-\Gamma_{j}\right), \\
& \bar{R}_{N}=\sum_{j=2}^{N-1} c_{j} J^{\prime \prime}\left(\bar{\Omega}_{j} \hat{\xi}_{j}+\left(1-\bar{\Omega}_{j}\right) \Gamma_{j}\right)\left(\hat{\xi}_{j}-\Gamma_{j}\right)^{2},
\end{aligned}
$$

where the rv's $\bar{\Omega}_{j}$ satisfy $0 \leq \bar{\Omega}_{j} \leq 1$. If we now are able to show e.g. that $E\left|\bar{R}_{N}\right|=\mathrm{o}(1)$ and $E \bar{Q}_{N}^{2}=\mathrm{o}(1)$, then (2.5) will follow.

We shall first make some remarks about $\bar{R}_{N}$. From (2.7) it is evident that we shall have to impose certain conditions on the derivatives of $J$ involved. It is tempting to require simply that $J^{\prime \prime}$ is bounded. Then, as $\left|c_{j}\right|=\mathrm{O}\left(N^{-1 / 2}\right)$, showing that $E\left|\bar{R}_{N}\right|=\mathrm{o}(1)$ in view of $(2.7)$ boils down to showing that $\sum_{j=2}^{N-1} E\left(\hat{\xi}_{j}-\Gamma_{j}\right)^{2}=$ 
$\mathrm{o}\left(N^{-1 / 2}\right)$. As typically $\left(\hat{\xi}_{j}-\Gamma_{j}\right)=\mathrm{O}_{P}\left(j^{-1 / 2}+(N-j)^{-1 / 2}\right)$, this should not be too much of a problem. However, boundedness of the second derivative would rule out the choice $J=\left(\Phi^{-1}\right)^{2}$. But this choice corresponds to the normal case, which motivated our approach. Hence we shall have to work with a substantially weaker condition, for example of the following nature. Define for $s>0$ and $0<t<1$ the function $r_{s}(t)$ by $r_{s}(t)=\{t(1-t)\}^{-s}$, and require that

$$
J^{\prime \prime}(t)=\mathrm{O}\left(r_{2+\alpha}(t)\right)
$$

for some sufficiently small, but nonnegative $\alpha$ (cf. Albers, Bickel and van Zwet (1976) and more in particular Does (1982) for similar conditions). The choice $s=2+\alpha$ stems from the fact that for $J=\left(\Phi^{-1}\right)^{2}$ the minimal value of $\alpha$ such that (2.8) holds is precisely 0.

Unfortunately, using (2.8) rather than boundedness of $J^{\prime \prime}$ effectively ruins the above indicated straightforward approach towards showing that $E\left|\bar{R}_{N}\right|=\mathrm{o}(1)$. A considerably more delicate analysis is required to handle the case where both $J$ and $\hat{\xi}_{j}-\Gamma_{j}$ cause boundary effects. The interested reader is referred to the appendix. Here we merely present the result in the theorem below.

Next we consider $\bar{Q}_{N}$. Clearly, even if $J^{\prime \prime}$ (or $J^{\prime}$ ) were bounded, the direct approach sketched above would never give more in this case than $E\left|\bar{Q}_{N}\right|=$ $\mathrm{O}\left(N^{-1 / 2} \sum_{j=2}^{N-1} E\left|\hat{\xi}_{j}-\Gamma_{j}\right|\right)$, which is typically of order 1 , rather than o(1). This indicates that we need additional conditions here. It turns out that these are precisely the symmetry conditions on $f$ and $J$ mentioned earlier in connection with the test based on the $\tilde{X}_{j}$. Using these assumptions, we shall demonstrate in the appendix essentially that $E \bar{Q}_{N}^{2}=\mathrm{o}(1)$.

Combining the results from the appendix we arrive at our main result:

Theorem 2.1. Suppose that $m / N$ is bounded away from 0 and 1 , that the $c_{j}$ satisfy (2.1) and that $f^{\prime}$ is bounded. Moreover, assume that $f(-x)=f(x)$, $-\infty<x<\infty$, and $J(1-t)=J(t), 0<t<1$. If $E\left|X_{1}\right|^{n}<\infty$ for $n$ sufficiently large and $J$ satisfies (2.8) for $\alpha \geq 0$ sufficiently small, then (2.5) holds. If moreover $\lim _{t \rightarrow 0,1} t(1-t)\left|J^{\prime \prime}(t)\right| / J^{\prime}(t) \mid<2$, then (2.5) also holds for exact rather than approximate scores.

Proof. By Lemma A.2-A.4 we have that $E\left|R_{N}\right|=0$ (1) and by Lemma A.5-A.7 that $E Q_{N}^{2}=\mathrm{o}(1)$. Hence (A.5) implies that $E\left|\hat{T}_{N}-S_{N}\right|=\mathrm{o}(1)$, from which it follows through Lemma A.1 that $E\left|\hat{W}_{N}-V_{N}\right|=0(1)$. This in its turn gives that $E\left|\hat{W}_{N}-W_{N}\right|=\mathrm{o}(1)$, which in view of Lemma 2.1 shows that (A.5) holds. The last statement of the Theorem follows from Lemma A.8.

Remarks. 1. For $F=\Phi, J=\left(\Phi^{-1}\right)^{2}$, the conditions are satisfied.

2. It can be verified that the minimal value allowed for both $n$ and $\alpha^{-1}$ is about 20. Since this is quite large, it did not seem worthwhile to include the additional 
details needed to demonstrate this. Note that the large value is caused by the combined boundary effects of $J$ and $\hat{\xi}_{j}-\Gamma_{j}$. If $J^{\prime \prime}$ is bounded for example, then any $n>4$ will do.

\section{Appendix}

From Section 2 it follows that we shall be dealing with the situation where the $X_{j}, j=1, \ldots, N$, are independent $\mathrm{rv}$ 's with common $\mathrm{df} F$ and where the $\hat{X}_{j}$, $j=2, \ldots, N-1$, are as given by (1.5). From this last definition it is clear that for $j /(N+1)$ near 0 and 1 the difference $\hat{X}_{j}-X_{j}$ will not necessarily be small. Our first step will consist of showing that a sufficiently small fraction of such $\hat{X}_{j}$ can be omitted without changing the asymptotics. For some $\delta$ with $0<\delta<\frac{1}{2}$, introduce the following convention: if summation over some index, say $i$, is abbreviated to $\sum_{i}$, it will always be understood to mean

$$
\sum_{i=\beta_{N} N+1}^{\left(1-\beta_{N}\right) N}, \quad \text { with } \beta_{N}=N^{-1}\left[N^{1 / 2-\delta}\right]
$$

where $[y]$ means the integer part of $y$. Then we shall compare $\hat{W}_{N}$ and the $\hat{\xi}_{j}$ from (2.3) to

$$
\hat{T}_{N}=\sum_{j} c_{j} J\left(\hat{\psi}_{j}\right), \quad \hat{\psi}_{j}=\beta_{N}+N^{-1} \sum_{k} u\left(\hat{X}_{j}-\hat{X}_{k}\right) .
$$

Note that in going from $\hat{W}_{N}$ to $\hat{T}_{N}$, the $\hat{X}_{j}$ at the boundaries are removed in two respects: in the first place, only the standardized ranks $\hat{\psi}_{j}$ in the 'middle' are used. In the second place, in evaluating these ranks, only the $\hat{X}_{k}$ in the 'middle' are used. Moreover note that, whereas the $\hat{\xi}_{j}$ attain values in $\left[(N+1)^{-1}, 1-(N+1)^{-1}\right]$, the $\hat{\psi}_{j}$ attain values in $\left[\beta_{N}, 1-\beta_{N}\right]$. This reveals an additional advantage of using $\hat{T}_{N}$ : not only the boundary effects caused by the $\hat{X}_{j}$ are diminished, but also those due to $J$ (cf. Does (1982), p. 988).

Since we want to compare $\hat{W}_{N}$ to $W_{N}$ and/or $V_{N}$, we need to introduce counterparts for these statistics too. $T_{N}$ and the $\psi_{j}$ are obtained from (A.2) by removing the everywhere, while for $V_{N}$ we have (cf. also (2.6))

$$
S_{N}=\sum_{j} c_{j} J\left(\Lambda_{j}\right), \quad \Lambda_{j}=\beta_{N}+\left(1-2 \beta_{N}\right) \Gamma_{j} .
$$

In the next lemma we show that in order to prove that $E\left|\hat{W}_{N}-W_{N}\right|$ (or $E\left|\hat{W}_{N^{-}}-V_{N}\right|$ ) is o(1), it suffices to show the corresponding result for $\hat{T}_{N}$ and $T_{N}$ (or $\hat{T}_{N}$ and $\left.S_{N}\right)$.

Lemma A.1. Suppose that $m / N$ is bounded away from 0 and 1 , that the $c_{j}$ satisfy (2.1) and that $J$ satisfies (2.8) for some $0 \leq \alpha<\frac{1}{2}$. Let $\delta$ in (A.1) satisfy $\alpha<\delta<\frac{1}{2}$, then $E\left|\hat{T}_{N}-\hat{W}_{N}\right|, E\left|T_{N}-W_{N}\right|$ and $E\left|S_{N}-V_{N}\right|$ are all $\mathrm{o}(1)$. 
Proof. First we prove the result for $\hat{T}_{N}$. The argument for $T_{N}$ is identical. We have that

$$
\left|\hat{T}_{N}-\hat{W}_{N}\right| \leq\left(\sum_{j=2}^{N-1}-\sum_{j}\right)\left|c_{j}\right|\left|J\left(\hat{\xi}_{j}\right)\right|+\sum_{j}\left|c_{j}\right|\left|J\left(\hat{\psi}_{j}\right)-J\left(\hat{\xi}_{j}\right)\right| .
$$

From (2.8) it follows that $J^{\prime}(t)=\mathrm{O}\left(r_{1+\alpha}(t)\right)$ for $\alpha \geq 0$ and that $J(t)=\mathrm{O}\left(r_{\alpha}(t)\right)$ for $\alpha>0$ and $\mathrm{O}\left(\log r_{1}(t)\right)$ for $\alpha=0$. Hence the first term in (A.4) is of order $\beta_{N} N N^{-1 / 2} N^{\alpha} \log N$, which is o(1). The second term is slightly more complicated to handle. From (2.3) and (A.2) it follows that $\left|\hat{\psi}_{j}-\hat{\xi}_{j}\right| \leq \beta_{N}+N^{-1}=\mathrm{O}\left(\beta_{N}\right)$. Consequently,

$$
\sum_{j}\left|c_{j}\right|\left|J\left(\hat{\psi}_{j}\right)-J\left(\hat{\xi}_{j}\right)\right|=\mathrm{O}\left(\beta_{N} N^{-1 / 2} \sum_{j}\left|J^{\prime}\left(\Omega_{j} \hat{\psi}_{j}+\left(1-\Omega_{j}\right) \hat{\xi}_{j}\right)\right|\right),
$$

for certain rv's $\Omega_{j}$ with $0 \leq \Omega_{j} \leq 1$. As $r_{s}$ is convex, it follows that the second term in (A.4) is

$$
\begin{aligned}
\mathrm{O}\left(\beta_{N} N^{-1 / 2} \sum_{j}\left\{r_{1+\alpha}\left(\hat{\psi}_{j}\right)+r_{1+\alpha}\left(\hat{\xi}_{j}\right)\right\}\right) & =\mathrm{O}\left(\beta_{N} N^{-1 / 2} \sum_{j=2}^{N-1} r_{1+\alpha}\left(N^{-1} j\right)\right) \\
& =\mathrm{O}\left(\beta_{N} N^{1 / 2+\alpha}\right)=\mathrm{o}(1) .
\end{aligned}
$$

For $S_{N}$ we use that

$$
\begin{aligned}
& E\left|S_{N}-V_{N}\right|=\mathrm{O}( N^{-1 / 2}\left(\sum_{j=1}^{N}-\sum_{j}\right) E\left|J\left(\Gamma_{j}\right)\right| \\
&\left.+N^{-1 / 2} \sum_{j} E\left|J\left(\beta_{N}+\left(1-2 \beta_{N}\right) \Gamma_{j}\right)-J\left(\Gamma_{j}\right)\right|\right) \\
&=\mathrm{O}\left(N^{1 / 2} \beta_{N}+N^{1 / 2} \int_{0}^{1}\left|J\left(\beta_{N}+\left(1-2 \beta_{N}\right) t\right)-J(t)\right| \mathrm{d} t\right)=\mathrm{o}(1),
\end{aligned}
$$

since the integral involved is $O\left(\beta_{N}^{1-\alpha}\right)$, which follows e.g. by splitting the region of integration into $\left[\beta_{N}, 1-\beta_{N}\right]$ and its complement.

Hence if $\delta>\alpha$ in (A.1), we can in the sequel work with $\hat{T}_{N}, T_{N}$ and $S_{N}$, rather than with $\hat{W}_{N}, W_{N}$ and $V_{N}$. In analogy to (2.7) we consider the expansion $\hat{T}_{N}=$ $S_{N}+Q_{N}+R_{N}$, where

$$
\begin{aligned}
& Q_{N}=\sum_{j} c_{j} J^{\prime}\left(\Lambda_{j}\right)\left(\hat{\psi}_{j}-\Lambda_{j}\right), \\
& R_{N}=\sum_{j} c_{j}\left\{J^{\prime}\left(\Omega_{j} \hat{\psi}_{j}+\left(1-\Omega_{j}\right) \Lambda_{j}\right)-J^{\prime}\left(\Lambda_{j}\right)\right\}\left(\hat{\psi}_{j}-\Lambda_{j}\right) .
\end{aligned}
$$

First we shall consider the problem of proving that $E\left|R_{N}\right|=o(1)$. In Section 2 we saw that a straightforward attempt to show that $E\left|\bar{R}_{N}\right|=0$ (1) failed. Let us again apply a heuristic argument to find out whether we may expect to do better now that we have replaced $\bar{R}_{N}$ by $R_{N}$. Note that $R_{N}$ can also be written as

$$
\frac{1}{2} \sum_{j} c_{j} J^{\prime \prime}\left(\tilde{\Omega}_{j} \hat{\psi}_{j}+\left(1-\tilde{\Omega}_{j}\right) \Lambda_{j}\right)\left(\hat{\psi}_{j}-\Lambda_{j}\right)^{2} .
$$


Now typically $\left(\hat{\psi}_{j}-\Lambda_{j}\right)^{2}=\mathrm{O}_{P}\left(N^{-1} r_{1}\left(N^{-1} j\right)\right)$, and this observation, together with (2.8) and the convexity of $r_{s}$, leads to the conjecture that

$$
\begin{aligned}
E\left|R_{N}\right| & \sim N^{-3 / 2} \sum_{j} r_{2+\alpha}\left(N^{-1} j\right) \sim N^{-1 / 2} \int_{\beta_{N}}^{1-\beta_{N}} r_{2+\alpha}(t) \mathrm{d} t \\
& \sim N^{-1 / 2} \beta_{N}^{-1-\alpha} \sim N^{\alpha+\delta+\alpha \delta} .
\end{aligned}
$$

Consequently, replacing $\bar{R}_{N}$ by $R_{N}$ has helped, but it is still not good enough.

Hence we need an additional argument, and this will be based on the following observation: conditional on $X_{j}=x_{j}$, we typically have that

$$
\left(\hat{\psi}_{j}-\Lambda_{j}\right)^{2}=\mathrm{O}_{P}\left(N^{-1} r_{1}\left(N^{-1} j\right) / r_{1}\left(\gamma_{j}\right)\right),
$$

where $\gamma_{j}=F\left(x_{j}\right)$ (cf. (2.6)). Using such a result, we will be able to obtain a better bound on the rate of growth of e.g. $J^{\prime}\left(\Lambda_{j}\right)\left(\hat{\psi}_{j}-\Lambda_{j}\right)^{2}$ near 0 and 1 than before (cf. Albers, Bickel and van Zwet (1976) and Does (1982) for arguments of a similar nature). It will turn out that the improvement achieved indeed suffices to obtain the desired result $E\left|R_{N}\right|=\mathrm{o}(1)$. As a first step we state the following lemma:

Lemma A.2. Suppose that the conditions of Lemma A.1 hold. If

$$
\sum_{j}\left\{E r_{2+2 \alpha}\left(\Lambda_{j}\right)\left(\hat{\psi}_{j}-\Lambda_{j}\right)^{4}\right\}^{1 / 2}\left\{E r_{2+2 \alpha}\left(\hat{\psi}_{j}\right)\right\}^{1 / 2}=\mathrm{o}\left(N^{1 / 2}\right),
$$

then $E\left|R_{N}\right|=o(1)$ will hold.

Proof. For $0<u, v, w<1$ we have that

$$
\begin{aligned}
\left|J^{\prime}(w v+(1-w) u)-J^{\prime}(u)\right| & \leq\left|\int_{u}^{w v+(1-w) u}\right| J^{\prime \prime}(t)|\mathrm{d} t| \\
& \leq\left|\int_{u}^{v}\right| J^{\prime \prime}(t)|\mathrm{d} t|=\mathrm{O}\left(\left|r_{1+\alpha}(v)-r_{1+\alpha}(u)\right|\right) \\
& =\mathrm{O}\left(|v-u| r_{1+\alpha}(v) r_{1+\alpha}(u)\right) .
\end{aligned}
$$

Application of this result to $R_{N}$ in (A.5) shows that

$$
\left|R_{N}\right|=\mathrm{O}\left(\sum_{j}\left|c_{j}\right| r_{1+\alpha}\left(\hat{\psi}_{j}\right) r_{1+\alpha}\left(\Lambda_{j}\right)\left(\hat{\psi}_{j}-\Lambda_{j}\right)^{2}\right) \text {, }
$$

from which the assertion of the lemma follows immediately.

Next we obtain a conditional moment bound on $\hat{\psi}_{j}-\Lambda_{j}$.

Lemma A.3. Suppose that $f^{\prime}$ is bounded. Then, for each $0<\varepsilon<\frac{1}{2}$, there exists $n>0$ such that $E\left|X_{1}\right|^{n}<\infty$ implies that for each $s>0$,

$$
\begin{aligned}
& E\left(\left\{\left|\hat{\psi}_{j}-\Lambda_{j}\right|^{s}\right\}^{1 / s} \mid X_{j}=x_{j}\right) \\
& \quad=\mathrm{O}\left(N^{-1 / 2+\varepsilon} r_{1 / 2}\left(N^{-1} j\right) / r_{1 / 2}\left(\gamma_{j}\right)+N^{-1+\varepsilon} r_{1}\left(N^{-1} j\right)\right),
\end{aligned}
$$

where $\gamma_{j}=F\left(x_{j}\right)$. 
Proof. We shall write $\left(\hat{\psi}_{j}-\Lambda_{j}\right)=\left(\hat{\psi}_{j}-\psi_{j}\right)+\left(\psi_{j}-\Lambda_{j}\right)$ and prove the result for each of these two terms separately. First we consider the more difficult part, which clearly is $\left(\hat{\psi}_{j}-\psi_{j}\right)$. From (A.2) and (1.5) it follows that this term can be written as

$$
\begin{aligned}
& \hat{\psi}_{j}-\psi_{j}=N^{-1} \sum_{k \neq j} T_{j k}, \\
& T_{j k}=u\left(\varrho_{j}\left(X_{j}-\bar{X}_{j}\right)-\varrho_{k}\left(X_{k}-\bar{X}_{k}\right)\right)-u\left(X_{j}-X_{k}\right) .
\end{aligned}
$$

Consequently, for integer $s$ we obtain that

$$
\begin{aligned}
E\left(\left|\hat{\psi}_{j}-\psi_{j}\right|^{s} \mid X_{j}=x_{j}\right) & \leq E\left(N^{-1} \sum_{k \neq j}\left|\tau_{j k}\right|\right)^{s} \\
& =\mathrm{O}\left(N^{-s} \sum_{l=1}^{s} \sum_{k_{1} \neq \cdots \neq k_{i} \neq j} E \prod_{i=1}^{l}\left|\tau_{j k_{i}}\right|\right),
\end{aligned}
$$

where $\tau_{j k}$ is obtained by replacing $X_{j}$ everywhere in $T_{j k}$ by $x_{j}$.

To deal with the last term in (A.9) we proceed as follows: first assume for simplicity that either $k<j \leq m$ or $k>j>m$. Then $\bar{X}_{k}$ contains no $x_{j}$-term. As moreover $|u(a+x)-u(x)| \leq u(|a|-|x|)$ and $u(|a| x)=u(x)$ for all $a$ and $x$, we use in this situation that

$$
\left|\tau_{j k}\right| \leq u\left(\left|\varrho_{j}-\varrho_{k}\right|\left|X_{k}\right|+\varrho_{j}\left|\bar{X}_{j}\right|+\varrho_{k}\left|\bar{X}_{k}\right|-\varrho_{j}\left|x_{j}-X_{k}\right|\right) .
$$

Application of Markov's inequality gives that

$$
\begin{aligned}
P\left(\left|\bar{X}_{j}\right| \geq N^{-1 / 2+\varepsilon} r_{1 / 2-\varepsilon}\left(N^{-1} j\right)\right) & =\mathrm{O}\left(N^{-n \varepsilon} r_{n \varepsilon}\left(N^{-1} j\right)\right) \\
& =\mathrm{O}\left(\left(\beta_{N} N\right)^{-n \varepsilon}\right)=\mathrm{O}\left(N^{-p}\right),
\end{aligned}
$$

where $p$ can be made arbitrarily large by choosing $n$ sufficiently large for given $\varepsilon$. For $\left|\bar{X}_{k}\right|$ a similar result holds. Since $\left|\varrho_{j}-\varrho_{k}\right| \leq N^{-1}\left\{r_{1}\left(N^{-1} j\right)+r_{1}\left(N^{-1} k\right)\right\}$, it also follows that

$$
P\left(\left|\varrho_{j}-\varrho_{k}\right|\left|X_{k}\right| \geq N^{-1 / 2+\varepsilon}\left\{r_{1 / 2-\varepsilon}\left(N^{-1} j\right)+r_{1 / 2-\varepsilon}\left(N^{-1} k\right)\right\}\right)=\mathrm{O}\left(N^{-p}\right) .
$$

Finally note that $\frac{1}{2}<\varrho_{j}, \varrho_{k}<1$. These facts together with (A.10) lead to the conclusion that for $k_{1} \neq \cdots \neq k_{l}<j \leq m$ or $k_{1} \neq \cdots \neq k_{l}>j>m$,

$$
\begin{aligned}
E \prod_{i=1}^{l}\left|\tau_{j k_{i}}\right| \leq \prod_{i=1}^{l} P\left(\left|X_{k_{i}}-x_{j}\right| \leq\right. & \left.4 N^{-1 / 2+\varepsilon}\left\{r_{1 / 2}\left(N^{-1} j\right)+r_{1 / 2}\left(N^{-1} k_{i}\right)\right\}\right) \\
& +\mathrm{O}\left((l+1) N^{-p}\right) .
\end{aligned}
$$

That this result also holds for arbitrary $k_{1} \neq \cdots \neq k_{l}$ can be verified by a similar argument. Therefore we shall merely give an indication: let e.g. $j<k \leq m$, then write $\bar{X}_{k}=x_{j} /(k-1)+\bar{X}_{k}^{*}$, and use $\left\{\varrho_{j}+\varrho_{k} /(k-1)\right\}\left|x_{j}-X_{k}\right|$ rather than $\varrho_{j}\left|x_{j}-X_{k}\right|$ in (A.10). 
The result in (A.11) in its turn gives that

$$
\begin{aligned}
& N^{-l} \sum_{k_{1} \neq \cdots \neq k_{i} \neq j} E \prod_{i=1}^{\prime}\left|\tau_{j k_{i}}\right| \\
& \leq\left[N^{-1} \sum_{k \neq j} P\left(\left|X_{k}-x_{j}\right| \leq 4 N^{-1 / 2+\varepsilon}\left\{r_{1 / 2}\left(N^{-1} j\right)+r_{1 / 2}\left(N^{-1} k\right)\right\}\right)\right]^{l} \\
& \quad+\mathrm{O}\left(N^{-p}\right)
\end{aligned}
$$

The final argument needed is the following: from the assumption that $\left|f^{\prime}\right| \leq M$ for some finite $M$, it follows that $1-F(x)=\int_{x}^{\infty} f(y) \mathrm{d} y \geq f^{2}(x) / 2 M$ for all $x$. Likewise, $F(x) \geq f^{2}(x) / 2 M$ and we therefore obtain that $f^{2}(x)=\mathrm{O}(F(x)(1-F(x)))$, and hence that $f\left(x_{j}\right)=\mathrm{O}\left(\left(r_{1 / 2}\left(\gamma_{j}\right)\right)^{-1}\right)$. Consequently,

$$
P\left(\left|X_{k}-x_{j}\right| \leq|a|\right)=\mathrm{O}\left(|a| / r_{1 / 2}\left(\gamma_{j}\right)+a^{2}\right) .
$$

Combination of (A.9), (A.12) and (A.13) shows that (A.7) holds for $\left(\hat{\psi}_{j}-\psi_{j}\right)$.

It remains to show that (A.7) is also true for $\psi_{j}-\Lambda_{j}$. To this end we note that $N\left(\psi_{j}-\Lambda_{j}\right)$ is a binomial rv with parameters $\left(1-2 \beta_{N}\right) N$ and $\gamma_{j}$, which leads to the conclusion that $E\left(\left|N\left(\psi_{j}-\Lambda_{j}\right)\right|^{s} \mid X_{j}=x_{j}\right)=\mathrm{O}\left(N^{s / 2} / r_{s / 2}\left(\gamma_{j}\right)\right)$.

Now we shall use the result of this lemma to prove that the expectations occurring in (A.6) are sufficiently small to ensure that (A.6) is indeed true. Hence we shall show:

Lemma A.4. Suppose that $m / N$ is bounded away from 0 and 1 , that the $c_{j}$ satisfy (2.1) and that $f^{\prime}$ is bounded. If $E\left|X_{1}\right|^{n}<\infty$ for $n$ sufficiently large and moreover $J$ satisfies (2.8) for $\alpha \geq 0$ sufficiently small, then there exists $\delta>\alpha$ in (A.1) such that

$$
E\left|R_{N}\right|=\mathrm{o}(1) \text {. }
$$

Proof. Note that under the conditions above we can apply all previous lemma's. We shall repeatedly need a positive, but otherwise arbitrarily small constant. For simplicity we shall not write $\varepsilon_{1}, \varepsilon_{2}$, etc. for such constants, but instead use $\varepsilon$ as a generic constant. We begin with the first factor in (A.6). Application of (A.7) in this case gives that

$$
\begin{aligned}
& \left\{E r_{2+2 \alpha}\left(\Lambda_{j}\right)\left(\hat{\psi}_{j}-\Lambda_{j}\right)^{4}\right\}^{1 / 2} \\
& =\mathrm{O}\left(\left\{N^{-2+\varepsilon} r_{2}\left(N^{-1} j\right) \int_{0}^{1} r_{2+2 \alpha}\left(\beta_{N}+\left(1-2 \beta_{N}\right) t\right) / r_{2}(t) \mathrm{d} t\right.\right. \\
& \left.\left.\quad+N^{-4+\varepsilon} r_{4}\left(N^{-1} j\right) \int_{\beta_{N}}^{1-\beta_{N}} r_{2+2 \alpha}(t) \mathrm{d} t\right\}^{1 / 2}\right)
\end{aligned}
$$

Now the second term in this expansion differs from the first by a factor $\mathrm{O}\left(N^{-2+\varepsilon} r_{2}\left(N^{-1} j\right) \beta_{N}^{-1-2 \alpha}\right)=\mathrm{O}\left(N^{-2+\varepsilon} N^{(1 / 2+\delta)(3+2 \alpha)}\right)$, and this can be made o(1), 
since $\alpha$, and hence $\delta>\alpha$, can be chosen sufficiently small. It follows that

$$
\left\{E r_{2+2 \alpha}\left(\Lambda_{j}\right)\left(\hat{\psi}_{j}-\Lambda_{j}\right)^{4}\right\}^{1 / 2}=\mathrm{O}\left(N^{-1+\varepsilon} r_{1}\left(N^{-1} j\right)\right) \text {. }
$$

For the second factor in (A.6) observe that

$$
E r_{2+2 \alpha}\left(\hat{\psi}_{j}\right)=\sum_{k} r_{2+2 \alpha}\left(N^{-1} k\right) P\left(\hat{\psi}_{j}=N^{-1} k\right),
$$

which is of order

$$
\sum_{k}\left|r_{2+2 \alpha}\left(N^{-1} k\right)-r_{2+2 \alpha}\left(N^{-1}(k+1)\right)\right|\left\{P\left(\hat{\psi}_{j} \leq N^{-1} k\right) P\left(\hat{\psi}_{j} \geq 1-N^{-1} k\right)\right\} .
$$

To deal with this expression, we observe for all $\zeta_{N}$ and $q>0$ that

$$
P\left(\hat{\psi}_{j} \leq N^{-1} k\right) \leq P\left(\psi_{j} \leq N^{-1} k+\zeta_{N}\right)+\zeta_{N}^{-q} E\left|\hat{\psi}_{j}-\psi_{j}\right|^{q} .
$$

Since $N \psi_{j}$ is uniform on $\left\{\beta_{N} N+1, \beta_{N} N+2, \ldots,\left(1-\beta_{N}\right) N\right\}$, this leads to

$$
P\left(\hat{\psi}_{j} \leq N^{-1} k\right)=\left(N^{-1} k-\beta_{N}\right) /\left(1-2 \beta_{N}\right)+\mathrm{O}\left(\zeta_{N}+\zeta_{N}^{-q} E\left|\hat{\psi}_{j}-\psi_{j}\right|^{q}\right) .
$$

This in its turn gives

$$
P\left(\hat{\psi}_{j} \leq N^{-1} k\right)-\left(N^{-1} k-\beta_{N}\right) /\left(1-2 \beta_{N}\right)=\mathrm{O}\left(\left\{E\left|\hat{\psi}_{j}-\psi_{j}\right|^{q}\right\}^{1 /(q+1)}\right)
$$

by choosing $\zeta_{N}$ appropriately. In view of Lemma A.3 we then arrive at

$$
P\left(\hat{\psi}_{j} \leq N^{-1} k\right)=\left(N^{-1} k-\beta_{N}\right) /\left(1-2 \beta_{N}\right)+\mathrm{O}\left(N^{-1 / 2+\varepsilon} r_{1 / 2}\left(N^{-1} j\right)\right),
$$

where the factor $q /(q+1)$ has been omitted since $q$ can be chosen arbitrarily large. Application of (A.17) and of the fact that

$$
\left|r_{s}\left(N^{-1} k\right)-r_{s}\left(N^{-1}(k+1)\right)\right|=\mathrm{O}\left(N^{-1} r_{s+1}\left(N^{-1} k\right)\right)
$$

to (A.16) now leads to the conclusion that

$$
\begin{aligned}
& E r_{2}+2 \alpha\left(\hat{\psi}_{j}\right) \\
& \quad=\mathrm{O}\left(N^{-1} \sum_{k} r_{2+2 \alpha}\left(N^{-1} k\right)+N^{-3 / 2+\varepsilon} r_{1 / 2}\left(N^{-1} j\right) \sum_{k} r_{3+2 \alpha}\left(N^{-1} k\right)\right) \\
& \quad=\mathrm{O}\left(\beta_{N}^{-1-2 \alpha}+N^{-1 / 2+\varepsilon} r_{1 / 2}\left(N^{-1} j\right) \beta_{N}^{-2-2 \alpha}\right) .
\end{aligned}
$$

For $\delta$ sufficiently small, the first term is of a smaller order than the second. Hence we have that

$$
\left\{E r_{2+2 \alpha}\left(\hat{\psi}_{j}\right)\right\}^{1 / 2}=\mathrm{O}\left(N^{1 / 4+\varepsilon} r_{1 / 4}\left(N^{-1} j\right)\right) .
$$

Together (A.15) and (A.18) show that the expression in (A.6) is of order

$$
\mathrm{O}\left(N^{-3 / 4+\varepsilon} \sum_{j} r_{5 / 4}\left(N^{-1} j\right)\right)=\mathrm{O}\left(N^{1 / 4+\varepsilon} \beta_{N}^{-1 / 4}\right)=\mathrm{O}\left(N^{3 / 8+\varepsilon}\right) \text {, }
$$

which is indeed $o\left(N^{1 / 2}\right)$. Hence, by application of Lemma A.2 the desired result (A.14) now follows. 
From the lemma above it is clear that (A.7) only just suffices to obtain the desired result. A simpler bound, independent of $j$, like

$$
\left\{E\left|\hat{\psi}_{j}-\Lambda_{j}\right|^{s}\right\}^{1 / s}=\mathrm{O}\left(N^{-1 / 2+\varepsilon} r_{1 / 2}\left(\beta_{N}\right)\right)=\mathrm{O}\left(N^{-1 / 4+\varepsilon}\right)
$$

would not have been strong enough. It is also evident that the minimal $n$ and $\alpha^{-1}$ allowed will be quite large. For this reason we have not bothered to give the more precise analysis here which would be needed to obtain these values explicitly. We just remark that for both quantities the minimal value will be about 20 . Incidentally, if $J^{\prime \prime}$ is bounded, then $n=4+\varepsilon$ will do.

By proving (A.14) in Lemma A.4, we have concluded our treatment of $R_{N}$. Next we turn to our second task in this appendix, which consists of proving that $E Q_{N}^{2}=$ $o(1)$ (cf. (A.5)). We shall begin by isolating the essential part of this problem in the following lemma.

Lemma A.5. Suppose that the conditions of Lemma A.4 hold. Then $E Q_{N}^{2}=\mathrm{o}(1)$ will hold if ( $c f .(\mathrm{A} .8))$

$$
N^{-2} E \sum_{i \neq j} c_{i} c_{j} J^{\prime}\left(\Lambda_{i}\right) J^{\prime}\left(\Lambda_{j}\right) \sum_{\substack{k \neq i \\ k \neq 1}} \sum_{i \neq j} T_{i k} T_{j l}=\mathrm{o}(1) .
$$

Proof. From (A.5) it follows that

$$
E Q_{N}^{2} \leq 2 E\left\{\sum_{j} c_{j} J^{\prime}\left(\Lambda_{j}\right)\left(\hat{\psi}_{j}-\psi_{j}\right)\right\}^{2}+2 E\left\{\sum_{j} c_{j} J^{\prime}\left(\Lambda_{j}\right)\left(\psi_{j}-\Lambda_{j}\right)\right\}^{2} .
$$

Since the $J^{\prime}\left(\Lambda_{j}\right)\left(\psi_{j}-\Lambda_{j}\right)$ are identically distributed, while moreover

$$
\sum_{i \neq j} \sum_{i} c_{j}=\left(\left(\sum_{j=1}^{N}-\sum_{j}\right) c_{j}\right)^{2}-\sum_{j} c_{j}^{2}=\mathrm{O}\left(N \beta_{N}^{2}+1\right)=\mathrm{O}(1)
$$

(cf. once more Does (1982), p. 988), it follows that the last term in (A.20) is $\mathrm{O}\left(\sum_{j} c_{j}^{2} E\left\{J^{\prime}\left(\Lambda_{j}\right)\right\}^{2}\left(\psi_{j}-\Lambda_{j}\right)^{2}\right)$. As $E\left(\left(\psi_{j}-\Lambda_{j}\right)^{2} \mid X_{j}=x_{j}\right)=\mathrm{O}\left(N^{-1} / r_{1}\left(\gamma_{j}\right)\right)$ this expression in its turn is

$$
\mathrm{O}\left(N^{-1} \int_{\beta_{N}}^{1-\beta_{N}} r_{2+2 \alpha}(t) / r_{1}(t) \mathrm{d} t\right)=\mathrm{O}\left(N^{-1+\varepsilon}\right)=\mathrm{o}(1)
$$

where again $\varepsilon$ is a generic arbitrarily small positive constant. Using (A.7) we can incidentally show in precisely the same way that $\mathrm{O}\left(\sum_{j} c_{j}^{2} E\left\{J^{\prime}\left(\Lambda_{j}\right)\right\}^{2}\left(\hat{\psi}_{j}-\psi_{j}\right)^{2}\right)=\mathrm{o}(1)$.

To prove (A.19) it thus remains to show that

$$
E\left(N^{-2} \sum_{i \neq j} c_{i} c_{j} J^{\prime}\left(\Lambda_{i}\right) J^{\prime}\left(\Lambda_{j}\right) \sum_{k \neq i, j} T_{i k} T_{j k}\right)=\mathrm{o}(1) .
$$


As $\left|\sum_{k \neq i, j} T_{i k} T_{j k}\right| \leq \sum_{k \neq j}\left|T_{j k}\right|$ we obtain in view of Lemma A.3 (see in particular (A.9)) that

$$
\begin{aligned}
& \left|c_{i} c_{j} E J^{\prime}\left(\Lambda_{i}\right) J^{\prime}\left(\Lambda_{j}\right) \sum_{k \neq i, j} T_{i k} T_{j k}\right| \\
& \quad=\mathrm{O}\left(\left\{\mathrm{E}\left|J^{\prime}\left(\Lambda_{i}\right)\right|^{s /(s-1)}\right\}^{2(s-1) / s}\left\{E\left(N^{-1} \sum_{k \neq j}\left|T_{j k}\right|\right)^{s}\right\}^{1 / s}\right) \\
& \quad=\mathrm{O}\left(N^{-1 / 2+\varepsilon_{1 / 2}} r\left(N^{-1} j\right)\right),
\end{aligned}
$$

from which the desired result follows.

The obvious way to deal with (A.19) seems to be to first evaluate the expectation of $T_{i k} T_{j l}$ conditional on all rv's involved, except $X_{k}$ and $X_{l}$. However, here the complication arises that $X_{k}$ and $X_{l}$ may also occur in $\bar{X}_{i}, \bar{X}_{k}, \bar{X}_{j}$, and $\bar{X}_{l}$. To overcome this difficulty, we shall carry out the following program: we replace everywhere in $\bar{X}_{i}, \bar{X}_{k}, \bar{X}_{j}$ and $\bar{X}_{l}$ the rv's $X_{k}$ and $X_{l}$ by independent copies $\tilde{X}_{k}$ and $\tilde{X}_{l}$, and we denote the resulting averages by $\bar{X}_{i \mid k, l}, \bar{X}_{k \mid l}, \bar{X}_{j \mid k, l}$ and $\bar{X}_{l \mid k}$ respectively. Then we define

$$
T_{i k \mid l}=u\left(X_{i}-X_{k}-\bar{X}_{i \mid k, l}+\varrho_{i}^{-1} \varrho_{k} \bar{X}_{k \mid l}\right)-u\left(X_{i}-X_{k}\right),
$$

and similarly $T_{j l \mid k}$. Now it will suffice to show that the effect of replacing $T_{i k} T_{j l}$ by $T_{i k \mid l} T_{j l \mid k}$ is negligible and moreover that after such a replacement, (A.19) will hold. The first of these steps is contained in the following lemma.

Lemma A.6. Suppose that the conditions of Lemma A.4 hold. Then

$$
N^{-2} E \sum_{i \neq j} c_{i} c_{j} J^{\prime}\left(\Lambda_{i}\right) J^{\prime}\left(\Lambda_{j}\right) \sum_{\substack{k \neq i \\ k \neq l}} \sum_{\substack{1 \neq j \\ k \neq 1}}\left\{T_{i k} T_{j l}-T_{i k \mid l} T_{j l \mid k}\right\}=\mathrm{o}(1) .
$$

Proof. Since

$$
T_{i k \mid l} T_{j l \mid k}-T_{i k} T_{j l}=\left(T_{i k \mid l}-T_{i k}\right) T_{j l}+T_{i k \mid l}\left(T_{j l \mid k}-T_{j l}\right),
$$

we can split the expression in (A.22) into two parts and prove the result for each of these parts separately. As both terms are very similar, we shall restrict attention to the first one. From (A.8) and (A.21) it follows that

$$
\begin{aligned}
\left|T_{i k \mid l}-T_{i k}\right| \leq & u\left(2 N^{-1}\left\{r_{1}\left(N^{-1} i\right)+r_{1}\left(N^{-1} k\right)\right\}\left\{\left|X_{k}\right|+\left|\tilde{X}_{k}\right|+\left|X_{l}\right|+\left|\tilde{X}_{l}\right|\right\}\right. \\
& \left.-\left|\varrho_{i}\left(X_{i}-X_{k}-\bar{X}_{i \mid k, l}\right)+\varrho_{k} \bar{X}_{k \mid l}\right|\right) .
\end{aligned}
$$

Treating $T_{j l}$ as in Lemma A.3, we then obtain, since $P\left(\left|X_{k}\right| \geq N^{\varepsilon}\right)=\mathrm{O}\left(N^{-p}\right)$ with $p$ arbitrarily large, that

$$
\begin{aligned}
&\left.E\left(\mid T_{i k \mid l}-T_{i k}\right) T_{j l}|| X_{i}=x_{i}, X_{j}=x_{j}\right) \\
& \leq P\left(\left|\varrho_{i}\left(X_{k}-x_{i}+\bar{X}_{i \mid k, l}\right)-\varrho_{k} \bar{X}_{k \mid l}\right| \leq 8 N^{-1+\varepsilon}\left\{r_{1}\left(N^{-1} i\right)+r_{1}\left(N^{-1} k\right)\right\}\right. \\
& \wedge\left|X_{l}-x_{j}\right| \leq 4 N^{-1 / 2+\varepsilon}\left\{r_{1 / 2}\left(N^{-1} j\right)+r_{1 / 2}\left(N^{-1} l\right)\right\} \\
&\left.+2 N^{-1+\varepsilon}\left\{r_{1}\left(N^{-1} j\right)+r_{1}\left(N^{-1} l\right)\right\}\left\{\left|x_{i}\right|+\left|x_{j}\right|\right\} \mid X_{i}=x_{i}, X_{j}=x_{j}\right) \\
&+\mathrm{O}\left(N^{-p}\right),
\end{aligned}
$$


which implies that

$$
\begin{aligned}
& \left.N^{-2} \sum_{\substack{k \neq i \\
k \neq 1}} E\left(\mid T_{i k \mid l}-T_{i k}\right) T_{j l}|| X_{i}=x_{i}, X_{j}=x_{j}\right) \\
& =\mathrm{O}\left(N^{-3 / 2+\varepsilon} r_{1}\left(N^{-1} i\right)\left\{r_{1 / 2}\left(N^{-1} j\right)+N^{-1 / 2}\left(\left|x_{i}\right|+\left|x_{j}\right|\right) r_{1}\left(N^{-1} j\right)\right\}\right) .
\end{aligned}
$$

From this result it readily follows that the desired expectation is $\mathrm{O}\left(N^{-1 / 2+\varepsilon}\right)=$ $o(1)$.

The second and final step is:

Lemma A.7. Suppose that the conditions of Lemma A.4 hold. Moreover, assume that

$$
f(-x)=f(x), \quad-\infty<x<\infty, \quad J(1-t)=J(t), \quad 0<t<1 .
$$

Then (A.19) holds if $T_{i k} T_{j l}$ is replaced by $T_{i k \mid l} T_{j l \mid k}$.

Proof. We have that

$$
\begin{aligned}
E\left(T_{i k \mid l}\right. & \left.T_{j l \mid k} \mid X_{i}=x_{i}, X_{j}=x_{j}\right) \\
= & E\left(\left\{F\left(x_{i}-\bar{X}_{i}+\varrho_{i}^{-1} \varrho_{k} \bar{X}_{k}\right)-F\left(x_{i}\right)\right\}\right. \\
\quad & \left.\quad \times\left\{F\left(x_{j}-\bar{X}_{j}+\varrho_{j}^{-1} \varrho_{l} \bar{X}_{l}\right)-F\left(x_{j}\right)\right\} \mid X_{i}=x_{i}, X_{j}=x_{j}\right) \\
= & E\left(\left(\varrho_{i}^{-1} \varrho_{k} \bar{X}_{k}-\bar{X}_{i}\right)\left(\varrho_{j}^{-1} \varrho_{l} \bar{X}_{l}-\bar{X}_{j}\right) \mid X_{i}=x_{i}, X_{j}=x_{j}\right) \\
+ & \mathrm{O}\left(E\left(\left(\bar{X}_{k}^{2}+\bar{X}_{i}^{2}\right)\left(\bar{X}_{l}^{2}+\bar{X}_{j}^{2}\right) \mid X_{i}=x_{i}, X_{j}=x_{j}\right)\right) .
\end{aligned}
$$

Since (A.23) implies that e.g. $E J^{\prime}\left(\Lambda_{j}\right) f\left(X_{j}\right)=0$, the result now follows by straightforward computation.

This concludes our task of proving that $E Q_{N}^{2}=\mathrm{o}(1)$. The last point we have to deal with is that of showing that the results obtained continue to hold if we replace our approximate scores by exact scores (cf. (1.2)). We have:

Lemma A.8. Suppose that the $c_{j}$ satisfy (2.1), that $J$ satisfies (2.8) for some $0 \leq \alpha<\frac{1}{2}$ and that moreover

$$
\lim _{t \rightarrow 0,1} t(1-t)\left|\frac{J^{\prime \prime}(t)}{J^{\prime}(t)}\right|<2 .
$$

Then all previous lemma's continue to hold if exact rather than approximate scores are used. 
Proof. According to Albers, Bickel and van Zwet (1976), p. 152, we have under (A.24) that

$$
\left|E J\left(U_{j: N}\right)-J\left((N+1)^{-1} j\right)\right|=\mathrm{O}\left(N^{-1} / r_{1}\left(N^{-1} j\right)+N^{-1}\left|J^{\prime}\left((N+1)^{-1} j\right)\right|\right) .
$$

Consequently,

$$
\begin{aligned}
\left.N^{-1 / 2} \sum_{j=1}^{N} \mid E J\left(U_{j: N}\right)-J\left((N+1)^{-1} j\right)\right) \mid & =\mathrm{O}\left(N^{-3 / 2} \sum_{j=1}^{N}\left|J^{\prime}\left((N+1)^{-1} j\right)\right|\right) \\
& =\mathrm{O}\left(N^{-1 / 2+\alpha}\right)=\mathrm{o}(1),
\end{aligned}
$$

from which the desired result follows.

\section{References}

Albers, W., P.J. Bickel and W.R. van Zwet (1976). Asymptotic expansions for the power of distributionfree tests in the one-sample problem. Ann. Statist. 4, 108-156.

Cramér, H. (1946). Mathematical Methods of Statistics. Princeton University Press.

Does, R.J.M.M. (1982). Berry-Esséen Theorems for simple linear rank statistics under the null hypothesis. Ann. Probab. 10, 982-991.

Hájek, J. (1968). Asymptotic normality of simple linear rank statistics under alternatives. Ann. Math. Statist. 39, 325-346.

Hájek, J. (1970). Miscellaneous problems of rank test theory. In: M.L. Puri, Ed., Nonparametric Techniques in Statistical Inference. University Press, Cambridge, 3-13.

Hájek, J. and Z. Šidák (1967). Theory of Rank Tests. Academic Press, New York.

Kendall, M.G. and A. Stuart (1969). The Advanced Theory of Statistics, Vol. I, 3rd edition. Griffin, London.

Lehmann, E.L. (1975). Nonparametrics: Statistical Methods Based on Ranks. Holden-Day, San Francisco, CA.

Raghavachari, M. (1965). The two-sample scale problem when locations are unknown. Ann. Math. Statist. 36, 1236-1242.

Sukhatme, B. (1958). Testing the hypothesis that two populations differ only in location. Ann. Math. Statist. 29, 60-78. 